\title{
Activité Anti-Inflammatoire Et Études Phytochimiques De L'extrait Aqueux Des Écorces Distemonanthus Benthamianus Baill. (Caesalpiniaceae : Leguminosae - Caesalpinioideae)
}

Kouadio Kouakou John, Doctorant

Ouattara-Soro Fatou Shcherazade, Professeur

Abizi Georges, Doctorant

Zougrou N'guessan Ernest, Docteur

Kouakou Koffi Roger, Docteur

Begbin Kouassi Emile, Doctorant

Kplé Tatiana Kangah Mireille, Doctorant

Kablan Kassi Jean-Jacques, Doctorant

Koffi Severin, Doctorant

UFR Biosciences,

Université Felix Houphouët Boigny, Abidjan, Côte d'Ivoire

Doi:10.19044/esj.2021.v17n7p74

Submitted: 05 December 2020

Accepted: 15 January 2021

Published: 28 February 2021
Copyright 2021 Author(s)

Under Creative Commons BY-NC-ND

4.0 OPEN ACCESS

Cite As:

John K.K., Fatou Shcherazade O-S., Georges A., N'guessan Ernest Z., Koffi Roger K., Kouassi Emile B., Kangah Mireille K.T., Jean-Jacques K.K. \& Severin K. (2021). Activité Anti-Inflammatoire Et Études Phytochimiques De L'extrait Aqueux Des Écorces Distemonanthus Benthamianus Baill. (Caesalpiniaceae : Leguminosae - Caesalpinioideae). European Scientific Journal, ESJ, 17(7), 74. https://doi.org/10.19044/esj.2021.v17n7p74

\section{Résumé}

Distemonanthus benthamianus est une plante utilisée en médecine traditionnelle dans certains pays Africains tels que : la Côte d'Ivoire, le Ghana, le Cameroun etc. Plusieurs études ont été réalisées relatives à son effet sur les activités antioxydante, antitumorale, antituberculeuse, bactéricide, antivirale. L'objectif de ce travail a été d'évaluer in vivo l'activité anti-inflammatoire de l'extrait total aqueux des écorces de cette plante. Pour atteindre cet objectif, le screening phytochimique a été réalisé afin de déterminer les métabolites secondaires présentent dans l'extrait de la plante. L'activité antiinflammatoire a été évalué par l'ingestion de la carragénine 1\% au niveau l'aponévrose plantaire des rats Wistars. Le screening phytochimique a révélé 
la présence de tanins, polyphénols, flavonoïdes, saponosides, de triterpènes et d'alcaloïdes Les résultats de l'activité anti-inflammatoire ont montré que le diclofénac et l'extrait aqueux de la plante ont atteint leur activité maximale d'inhibition de l'œdème à la 3ème heure avec une inhibition de $52.88 \%$, et $44.95 \%, 60.03 \%$ et 70.19 aux doses de 200,400 et $800 \mathrm{mg} / \mathrm{kg} / \mathrm{pc}$ respectivement. Quant à la prednisone, il a atteint son activité maximale de $26.66 \%$ à la 4ème heure. L'inhibition de l'œdème des pattes des rats par l'extrait de la plante montre ainsi les propriétés anti-inflammatoires de cet extrait. L'utilisation de cette plante comme un anti-inflammatoire en milieu naturel serait donc justifiée.

Mots clés : Edème, Anti-Inflammatoire, Distemonanthus Benthamianus

Anti-Inflammatory Activity and Phytochemical Studies of the Aqueous Extract of the Bark Distemonanthus Benthamianus Baill. (Caesalpiniaceae: Leguminosae - Caesalpinioideae)

Kouadio Kouakou John, Doctorant

Ouattara-Soro Fatou Shcherazade, Professeur

Abizi Georges, Doctorant

Zougrou N'guessan Ernest, Docteur

Kouakou Koffi Roger, Docteur

Begbin Kouassi Emile, Doctorant

Kplé Tatiana Kangah Mireille, Doctorant

Kablan Kassi Jean-Jacques, Doctorant

Koffi Severin, Doctorant

UFR Biosciences,

Université Felix Houphouët Boigny, Abidjan, Côte d'Ivoire

Abstract

Distemonanthus benthamianus is a plant used in traditional medicine in some African countries such as: Ivory Coast, Ghana, Cameroon etc. Several studies have been carried out relating to its effect on antioxidant, anti-tumor, anti-tuberculosis, bactericidal and antiviral activities. The objective of this work was to evaluate in vivo the anti-inflammatory activity of the total aqueous extract of the bark of this plant. To achieve this goal, phytochemical screening was carried out to determine the secondary metabolites present in the plant extract. Anti-inflammatory activity was assessed by ingestion of $1 \%$ carrageenan in the plantar fascia of Wistars rats. The phytochemical screening revealed the presence of tannins, polyphenols, flavonoids, saponosides, 
triterpenes and alkaloids The results of anti-inflammatory activity showed that diclofenac and the aqueous extract of the plant reached their maximum activity $\mathrm{d}$ inhibition of edema at the 3rd hour with an inhibition of $52.88 \%$, and $44.95 \%, 60.03 \%$ and 70.19 at doses of 200,400 and $800 \mathrm{mg} / \mathrm{kg} / \mathrm{bw}$ respectively. As for prednisone, it reached its maximum activity of $26.66 \%$ at the 4th hour. The inhibition of rat paw edema by the plant extract thus demonstrates the anti-inflammatory properties of this extract. The use of this plant as an anti-inflammatory in a natural environment would therefore be justified.

Keywords: Edema, Anti-Inflammatory, Distemonanthus benthamianus

\section{Introduction}

L'inflammation est un mécanisme de défense de l'organisme contre les agressions d'origine physique, chimique, biologique ou infectieuse, indispensable à son intégrité (Cheriti et al., 2016). Cette réponse immunitaire protectrice peut être parfois néfaste du fait de l'agressivité de l'agent pathogène, de sa persistance, des anomalies de régulation et de production des cellules intervenant dans l'inflammation (Weill et $a l, 2003$ ). Ces processus inflammatoires sont impliqués dans l'apparition d'un grand nombre de pathologies humaines tel que l'arthrite, le diabète, l'asthme, les allergies et le cancer (Viladomiu et al., 2016). L'inflammation et ses pathologies associées constituent de plus en plus un problème majeur de santé tant par la majorité de personnes qui en souffrent que aussi par les différentes formes sous lesquelles elles se manifestent. Leur traitement est souvent basé sur l'apport des anti-inflammatoires, non stéroïdiens (AINS) et des glucocorticoïdes. Ces molécules présentent des effets secondaires néfastes à l'organisme surtout en cas d'utilisation à longue durée, particulièrement dans le traitement des inflammations chroniques (Chiolero et al., 2000).

La prise des anti-inflammatoires présente souvent des risques gastrointestinaux (ulcère gastroduodénaux, perforation, sténose), des risques rénaux tels que l'insuffisance rénale aiguë et parfois des complications cardiaques (Soubrier et al., 2013). La recherche de nouvelles molécules médicales sans risques d'effets secondaires s'avère indispensable pour les traitements des sujets. C'est pourquoi, l'accent est de plus en plus mis sur la recherche de nouvelles molécules douées d'activités anti-inflammatoires dans les plantes médicinales. Par ailleurs, très peu d'étude sur l'activité anti-inflammatoire de Distemonanthus benthamianus ont été réalisées. C'est donc dans ce contexte que s'inscrit ce présent travail de recherche dont l'objectif consiste à évaluer les activités anti-inflammatoires et phytochimiques de l'extrait aqueux des écorces de Distemonanthus benthamianus, espèce de la sous-tribu de 
Dialiinae, sous-famille de Caesalpinioideae, famille de Fabaceae (APG IV, 2016).

Cette espèce est un arbre de taille moyenne à grande, atteignant $40 \mathrm{~m}$ de haut. La surface de l'écorce est lisse, parfois orange à rouge et devenant vert pâle à jaunâtre, se desquamant par grandes plaques irrégulières, écorce interne fine, fibreuse, crème à orange ou rose-brun, poisseuse ; cime ombelliforme, assez ouverte. Ses rameaux sont pubescentes courtes mais rapidement glabres, pourpres. Les feuilles sont alternes, composées imparipennées. L' inflorescence est cyme axillaire ayant l'apparence d'une panicule, atteignant $10(-30) \mathrm{cm}$ de long, à poils courts à des fleurs sont bisexuées, légèrement zygomorphes

(Distemonanthus_benthamianus_PROTA, 2019).

Les fruits sont des gousses elliptiques aplaties avec des graines elliptiques aplaties (CIRAD Forestry Department, 2009). L'espèce s'étend de la Guinée et de la Sierra Leone jusqu'au Cameroun, et vers le sud jusqu'au Gabon et au Congo méridional (Aiyegoro, et al., 2008). Cette plante est utilisée en médecine traditionnelle contre les infections de la peau, buccodentaires et urogénitales, les maladies inflammatoires telles que les hépatites, le rhumatisme, les bronchites et les troubles sanguin (Nguelefack et al., 2005 ; Akendengué et Louis, 1994).

\section{Matériel et méthodes \\ Matériel végétal}

Le matériel végétal comprend des écorces de la tige de Distemonanthus benthamianus (legumineae) récoltés dans la ville de Yakasse-Mé dans la région de la Mé et situé à $15 \mathrm{~km}$ de la ville d'Abidjan, Côte d'Ivoire. Les écorces ont été récoltées dans les mois de novembre et décembre 2018 et identifié au Jardin botanique de l'Université Felix Houphouët Boigny de Côte d'Ivoire. Ce spécimen a été répertorié dans l'index herbarium du centre floristique de Côte d'Ivoire sous le numéro 12473.

\section{Matériel animal}

Les rats Albinos de souche Wistar, mâles et femelles ont été élevés à l'animalerie de l'Ecole Nationale Supérieur d'Abidjan (ENS) où la température moyenne varie entre $25-26^{\circ} \mathrm{C}$, avec un cycle photopériodique de 12 heures de lumière/obscurité. Les rats sont logés dans des cages en plastique et ont été nourrit avec des granulés fournis par FACI (Société de Fabrication d'Aliments Composés Ivoiriens) additionnés à des pains secs provenant des boulangeries environnantes. Ils ont reçu comme eau de boisson de l'eau de robinet dans des biberons. La litière utilisée est la sciure, renouvelée deux fois par semaine pour assurer le bon état hygiénique des animaux. 


\section{Méthodes}

\section{Préparation de l'extrait}

Les écorces de Distemonanthus benthamianus ont été nettoyées et séchées à l'abri du soleil pendant quatre semaines. Elles ont été pulvérisées à l'aide d'un broyeur électrique. L'extrait aqueux de la plante a été obtenu à partir de $100 \mathrm{~g}$ de poudre dissous dans $1000 \mathrm{ml}$ d'eau distillée et homogénéisée à l'aide d'un mixeur pendant 15 minutes à la température ambiante à une vitesse de $3000 \mathrm{tr} / \mathrm{min}$. Après une période de décantation pendant 3 minutes, le surnageant a été filtrée sur du tissu blanc puis trois fois sur du coton hydrophile. Le filtrat a été séché dans une étuve de type Med center venticell à $55^{\circ} \mathrm{C}$ pendant 48 heures afin d'obtenir l'extrait sec.

\section{Étude du criblage phytochimique}

Criblages phytochimiques par la méthode de coloration en tube. Les analyses tri phytochimiques ont été réalisées au laboratoire de pharmacognosie de l'UFR des Sciences Pharmaceutiques.

Les tests phytochimiques préliminaires ont été réalisés sur les extraits aqueux d'écorce de D. benthamianus selon les méthodes décrites par Trease et Evan (2002).

Les flavonoïdes ont été caractérisés par la réaction dite à la cyanidine, les tanins catéchiques par le réactif de STIASNY (Formol $30 \%$, HCL concentré : $1 / 0,5)$, les tanins galliques par la réaction du réactif de STIASNY suivi du test au chlorure ferrique à $2 \%\left(\mathrm{FeCl}_{3}\right.$ à $\left.2 \%\right)$ et les polyphénols par le test de chlorure ferrique à $2 \%$. Le test de LIEBERMANN a permis de caractériser les polyterpènes et stérols. La caractérisation des saponosides est basée sur l'apparition d'une mousse après agitation de l'extrait. Les réactifs de DRAGENDORFF et BOUCHARDAT ont été utilisés pour la recherche des alcaloïdes. Quant à la recherche des quinoniques, elle a été faite en utilisant le réactif de BORNTRAEGEN (ammoniaque dilué au 1/2) et Les saponines avec le test à la mousse.

\section{Tests de détections des composés phytochimiques par la méthode de Chromatographie sur couche mince (CCM)}

La CCM a été réalisée pour confirmer la présence des certains groupes chimiques déterminés par la méthode de Trease et Evans (2002) dans l'extrait aqueux de d'écorce de D. benthamianus. L'identification des phytocomposés par CCM a été faite suivant les méthodologies décrites par (Békro et al., 2007 ; Brou et $a l ., 2010)$. La solution à tester a été déposée à l'aide de capillaire sous forme de points sur les plaques chromatographiques (60 F254, supportaluminium, 20×20, Fluka-Silica gel/DC). Les plaques ont été par la suite introduites dans une cuve de développement contenant un développant (mélange de solvants organiques). Les différents développants utilisés sont 
l'Hexane/Acétate d'éthyle (5/1), le Chloroforme/Acetate d'èthyle (6/5), l'Hex/AcoEt/AcOOH (4/3/4/0) et le n-Butanolique/Hex/ChCl (2/1). La séparation a été stoppée lorsque le front du développement a été atteint.

Les chromatogrammes ont été révélés à l'aide des réactifs spécifiques et/ou appropriés appelé (révélateurs) en solution vaporisée. Les révélateurs utilisés étaient le réactif Liebermann-Bürchard, Godin, Dragendorff, solution de $\mathrm{KOH} \mathrm{5 \% ,} \mathrm{AlCl}_{3}, \mathrm{FeCl}_{3}$ ainsi que la solution de Vanille sulfurique. Les plaques ont été observées dans le visible et sous observation UV. Les colorations des spots observés dans les chromatogrammes ont été enregistrées.

\section{Activité anti-inflammatoire de l'extrait aqueux des écorces de Distemonanthus benthamianus}

L'induction de l'œdème de la patte postérieure du rat par la carragénine (1\%) a été effectuée selon la méthode de Winter et collaborateurs (1962). Des rats ayant un poids compris entre $130 \mathrm{~g}$ et $170 \mathrm{~g}$ ont été mis en jeun pendant 24 heures. Les rats ont été repartis dans 6 lots de 6 rats chacun. Le diamètre au temps $\mathrm{T}_{0}$ de la patte postérieure droite a été déterminé à l'aide d'un pied à coulisse numérique (Bukhari, 2013).

Les différents lots ont été constitués : Les témoins œdémateux ont reçu l'eau distillée, les deux lots de référence, ont reçu respectivement le Diclofenac Sodium (20 mg/kg/kg) et le Prednisone $(20 \mathrm{mg} / \mathrm{kg} / \mathrm{pc})$, les lots EADB1, EADB2, et EADB3 ont reçu respectivement l'extrait aux doses de $200,400,800 \mathrm{mg} / \mathrm{kg} / \mathrm{pc}$.

Une heure après administration par gavage des différents solutions, 0,1 $\mathrm{ml}$ de la solution de carragénine à $1 \%$, il a été injecté par voie cutanée au niveau de l'aponévrose plantaire de la patte arrière droite de chaque rat. L'évolution de l'œdème droite a été déterminé à $1 \mathrm{~h}, 2 \mathrm{~h}, 3 \mathrm{~h}, 4 \mathrm{~h}, 5 \mathrm{~h}, 6 \mathrm{~h}$ (Anupana et al., 2012) et 24 heure. L'importance de l'œdème a été appréciée par la détermination de la moyenne du pourcentage d'augmentation (\%AUG) du volume de la patte de rat suivant la formule :

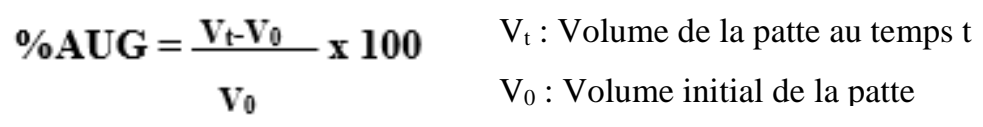

L'activité anti-inflammatoire a été aussi évaluée par le calcul du pourcentage d'inhibition (\% $\% \mathrm{INH})$ de l'œdème selon la formule :

\begin{tabular}{|c|c|c|c|}
\hline & $\%$ AUG $_{\text {та- }} \%$ AUG & & \% AUG $_{\text {TM }}$ : pourcentage augmentation des témoins \\
\hline & & $x>00$ & Traités \\
\hline
\end{tabular}




\section{Prelèvement du sang et analyse des marqueurs de l'inflammation}

Après 6 heures, le sang a été récupéré par la queue dans des tubes EDTA pour analyses hématologiques et des tubes secs pour les analyses immunobiochimiques à savoir la C-Protéine Réactive (CRP).

\section{Analyse statistique des résultats}

Les résultats ont été exprimés en moyenne accompagnées des erreurs standards sur la moyenne (Moyenne $\pm \mathrm{ESM}$ ). La répresentation graphique des donnés a été effectuée à partir du logiciel Graph Pad Prism 7.0 ( Microsoft USA). L'analyse statistique des résultats a été réalisée en utilisant l'analyse des variances (ANOVA ONE WAY). Les différences entre les moyennes ont été déterminées selon le test de comparaison de Dunnet, $\mathrm{P}<0,05$ est considéré comme significative.

\section{Résultats}

L'étude phytochimique de l'extrait aqueux de Distemonanthus benthamianus a permis d'obtenir les résultats présents dans le tableau I. Les résultats révèlent que les composés extractibles polaires sont majoritaires dans l'écorce de Distemonanthus benthamianus $s$. Il ressort de ce tableau que l'extrait aqueux d'écorce de $D$. benthamianus contient les polyphénols, les flavonoïdes, les polyterpènes et les tanins, des coumarines et les saponosides. Les alcaloïdes sont également présents dans les écorces de $D$. benthamianus à travers le test de DRAGENDORFF ; il a été également constaté l'absence des substances quinoniques.

Les résultats de la CCM présentés dans le tableau II révèlent la présence de coumarines, de flavonoïdes, de composés phénoliques, des triterpènes lupanes, des terpènes/stérols, des tanins et des alcaloïdes.

Tableau I. Etude phytochimique de l'extrait aqueux des écorces de D. benthamianus

\begin{tabular}{|c|c|c|}
\hline Composés recherchés & Procédure d'identification & $\begin{array}{c}\text { Extrait aqueux de } D . \\
\text { benthamianus }\end{array}$ \\
\hline Polyphénols & Fer (III)chlorure & + \\
\hline Tannin galliques & Fer (III)chlorure & + \\
\hline Flavonoïdes & Test de Shinoda & + \\
\hline Coumarine & Hydroxyde de potassium & + \\
\hline Stérols etTerpène & Liebermann-Buchard & + \\
\hline Saponines & Pouvoir aphrogène & + \\
\hline & Réactif de Dragendorff & - \\
\hline Alcaloïdes & Réactif de Bourchardart & + \\
\hline
\end{tabular}

Présence : + absence :- 
Tableau II. Identification des composés chimiques par le CCM

\begin{tabular}{|c|c|c|c|c|c|}
\hline & & & \multicolumn{2}{|c|}{ COLORATION } & \\
\hline Developants & Réactifs utilisés & Groupes chimiqies & Visible & UV/336 & REF \\
\hline \multirow{8}{*}{$\begin{array}{l}\text { Hexane/Acetate } \\
\text { d'ethyle }(5 / 1)\end{array}$} & \multirow[t]{2}{*}{$\mathrm{KOH}$} & \multirow[t]{2}{*}{ Coumarine } & & vert & 0.19 \\
\hline & & & & bleu & 0.25 \\
\hline & \multirow[t]{2}{*}{ APB } & \multirow[t]{2}{*}{ Coumarine } & & vert & 0.11 \\
\hline & & & & bLeu & 0.34 \\
\hline & GODIN & Sterols & violet & & 0.55 \\
\hline & \multirow[t]{2}{*}{ LB } & Sterols & brun & & 0.05 \\
\hline & & Triterpène lupane & orange & & 0.19 \\
\hline & VAN & Triterpène & violet & & 0.88 \\
\hline \multirow{11}{*}{$\begin{array}{c}\text { Chloroforle/ } \\
\text { Acetate d'ethyle } \\
\text { (6/5) }\end{array}$} & \multirow[t]{2}{*}{$\mathrm{KOH}$} & \multirow[t]{2}{*}{ Coumarine } & & jaune & 0.16 \\
\hline & & & & bleu & 0.40 \\
\hline & \multirow[t]{2}{*}{ APB } & \multirow[t]{2}{*}{ Coumarine } & & jaune & 0.11 \\
\hline & & & & bleu & 0.21 \\
\hline & \multirow[t]{3}{*}{ GODIN } & Terpène & rouge & & 0.09 \\
\hline & & Sterols & violet & & 0.58 \\
\hline & & Triterpène & vert & & 0.26 \\
\hline & \multirow[t]{2}{*}{$\mathrm{AlCl} 3$} & \multirow[t]{2}{*}{ Flavonoide } & & bleu & 0.14 , \\
\hline & & & & jaune & 0.30 \\
\hline & \multirow[t]{2}{*}{ DPPH } & Terpène & & jaune & 0.09 \\
\hline & & \multicolumn{2}{|c|}{ Activités antioxydante } & jaune pâle & 0.44 \\
\hline \multirow{8}{*}{$\begin{array}{c}\text { hex/CHCl3/ } \\
\text { AcoEth/ACOOH (4/3/4/0.5) }\end{array}$} & \multirow[t]{2}{*}{$\mathrm{KOH}$} & \multirow[t]{2}{*}{ Coumarine } & & vert & 0.13 \\
\hline & & & & bleu & 0.51 \\
\hline & APB & Coumarine & & vert & 0.53 \\
\hline & \multirow[t]{2}{*}{$\mathrm{FeCl} 3$} & Tanin & brun & & 0.53 \\
\hline & & Flavonoide & brun & & 0.53 \\
\hline & \multirow[t]{2}{*}{$\mathrm{AlCl} 3$} & \multirow[t]{2}{*}{ Flavonoide } & & bleu & 0.25 \\
\hline & & & & vert & 0.41 \\
\hline & DPPH & \multicolumn{2}{|c|}{ Tanin, Flavonoide } & jaune pâle & 0.56 \\
\hline \multirow[t]{4}{*}{$\mathrm{Hex} / \mathrm{CHCl} 3(2 / 1)$} & $\mathrm{KOH}$ & Coumarine & & vert & 0.70 \\
\hline & APB & Coumarine & & vert & 0.73 \\
\hline & \multirow[t]{2}{*}{$\mathrm{AlCl} 3$} & \multicolumn{2}{|c|}{ Composés phénolique } & rouge & 0.49 \\
\hline & & \multicolumn{2}{|c|}{ Flavonoide, composés phénoliques } & vert & 0.69 \\
\hline
\end{tabular}

\section{Evaluation des effets des différents produits sur l'œdème induite par la carragénine}

L'œdème a été induit au niveau de la patte postérieure gauche du rat. La variation de l'œdème a été appréciée une heure après l'administration des différents produits par voie orale. Les résultats obtenus ont été comparés à ceux du Diclofenac de Sodium (anti-inflammatoire non stéroïdien), en raison de $20 \mathrm{mg} / \mathrm{kg} / \mathrm{pc}$ et à ceux du prednisone (anti-inflammatoire stéroïdien). Les résultats des traités sont comparés à ceux des témoins œedémateux. L'injection de la carragénine $1 \%$ dans la patte postérieur gauche des rats témoins œdémateux provoque un œdème qui passe de $2.92 \pm 0.14 \mathrm{~mm}$ à $3.25 \pm 0.19 \mathrm{~mm}$ dans la première heure puis à $3.85 \pm 0.049 \mathrm{~mm}, 3.77 \pm 0.04$ et $3.67 \pm 0.05 \mathrm{~mm}$ 
après 4 heures, 6 heures et 24 heures respectivement (Figure 1). Ces valeurs correspondent à des pourcentages d'augmentation des œdèmes respectives de $11.44 \%, 32.13,30.41 \%$ et $26.66 \%$. Ces résultats montrent que l'œdème maximal a été atteint à la $4^{\mathrm{ème}}$ heure (Figure 2).

En présence d'extrait, les œedèmes des pattes ont atteint leurs maximums activités par la réduction de l'augmentation des œdèmes des pattes à la $6^{\text {ème }}$ heure pour les doses de 400 et $800 \mathrm{mg} / \mathrm{kg} / \mathrm{pc}$ avec $14,74 \%$ et $12,62 \%$ d'augmentation respectivement. Pour la dose de $200 \mathrm{mg} / \mathrm{kg} / \mathrm{pc}$, le pourcentage d'augmentation d'œdème maximal de $23.26 \%$ a été atteint à la $5^{\text {ème }}$ heure (Figure 3). Comparé aux témoins œémateux ces résultats sont considérablement significatifs pour EADB2 ( $\mathrm{p}<0.001)$ et EADB3 $(\mathrm{p}<0.0001)$. L'extrait a prévenu considérablement l'œdème des pattes des rats avec des pourcentages d'inhibition maximal de $32.63 \%$; $63.11 \%$ et $73.19 \%$ respectivement pour les lots EADB1, EADB2 et EADB3 à la troisième heure.

En présence de Diclofenac, il a été enregistré une augmentation moins importante du diamètre de la patte dont le maximum était de $17.48 \%$ au bout de la quatrième heure (Figure 3). La Figure 4 montre les pourcentages d'inhibition de l'œdème par le Diclofenac de sodium et l'extrait. Le Diclofenac a prévenu l'œdème dès la première heure (33.99\%) avec un maximal de $52.88 \%$ à la troisième heure après injection de la carragénine $1 \%$.

Les rats ayants reçu l'extrait aux doses de $800 \mathrm{mg} / \mathrm{kg} / \mathrm{pc}$ ont montré un pourcentage d'inhibition très prononcé par rapport au Diclofenac $(\mathrm{P}<0.01)$ au bout des 24 heures d'appréciation.

Pour la dose de $400 \mathrm{mg} / \mathrm{kg} / \mathrm{pc}$, il a été indiqué qui n'existe aucune différence significative ( $>>0.05)$ par rapport au Diclofenac. Cependant, le Diclofenac s'est montré plus efficace que l'extrait à la dose de $200 \mathrm{mg} / \mathrm{kg} / \mathrm{pc}(\mathrm{p}<0.05)$ (Figure 4).

La figure 5, indique que le lot qui a reçu la prednisone a montré une augmentation importante de l'œdème dont le maximum de $27.35 \%$ est atteint après la troisième heure. Ce qui correspond à un pourcentage d'inhibition de $25.58 \%$ (Figure 6). Le maximum de pourcentage d'inhibition de $26.16 \%$ est atteint à la $4^{\text {ème }}$ heure. Il a été constaté que son action sur l'inhibition est significativement faible $(\mathrm{p}<0.0001)$ par rapport à celle exercé par l'extrait aux doses de 400 et $800 \mathrm{mg} / \mathrm{kg} / \mathrm{pc}$ (EADB2, EADB3). Cependant, il n'existe pas de différence significative $(\mathrm{P}>0.05)$ entre le pourcentage d'inhibition de prednisone et l'extrait à la dose de $200 \mathrm{mg} / \mathrm{kg} / \mathrm{pc}$ (EADB1). L'action du Prednisone (AS) sur l'inhibition de l'augmentation de l'œdème est très faible par rapport au Diclofenac de Sodium (ANS) avec une différence significative, $\mathrm{p}<0.0001$. 

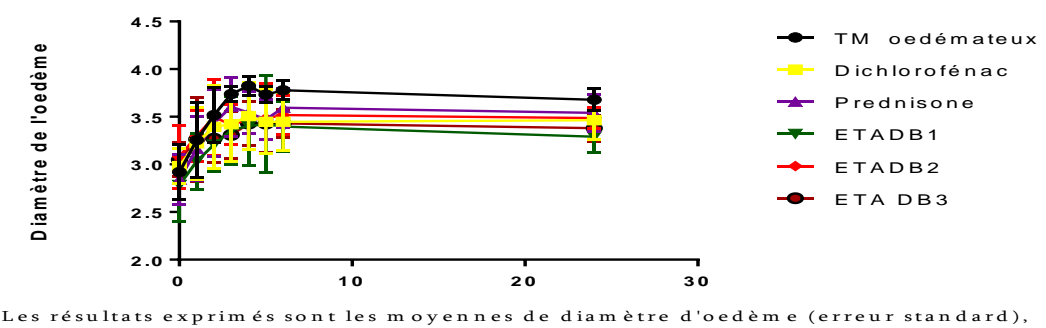

pas de différence significative par rapportau témoin oedémateux.

Figure 1. Variation de diamètre

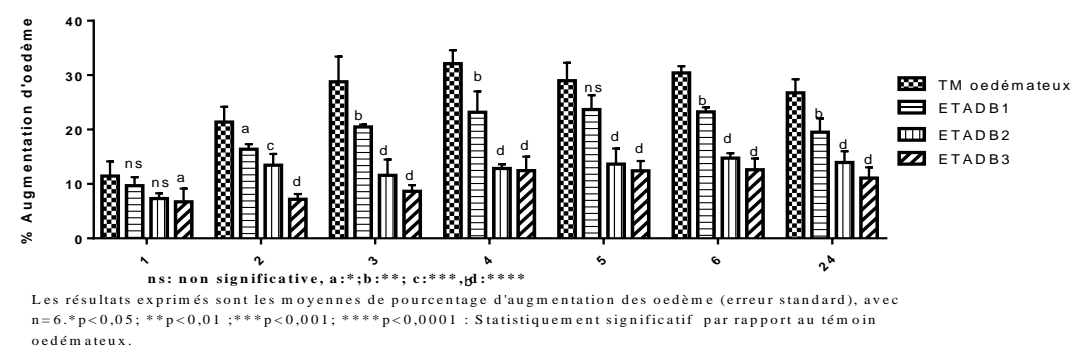

Figure 2. Pourcentage d'augmentation de 1'œdème

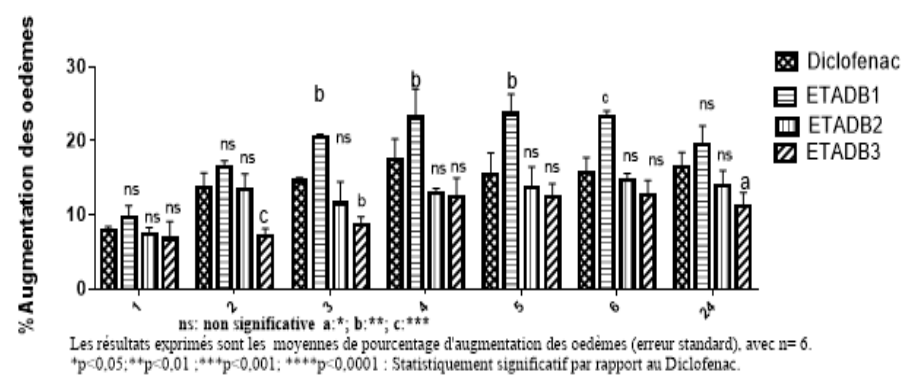

Figure 3. Pourcentage d'augmentation de l'œdème des lots ETADB et Diclofe

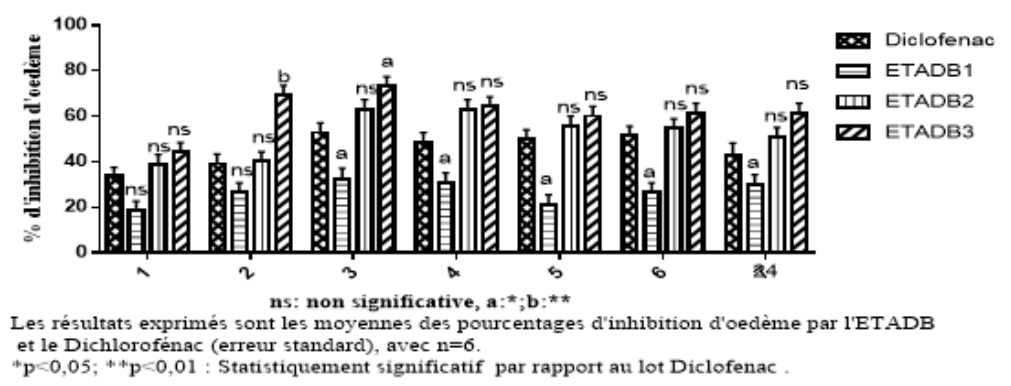

Figure 4. Effet d'inhibition de l'œdème par l'extrait et le Diclofénac 


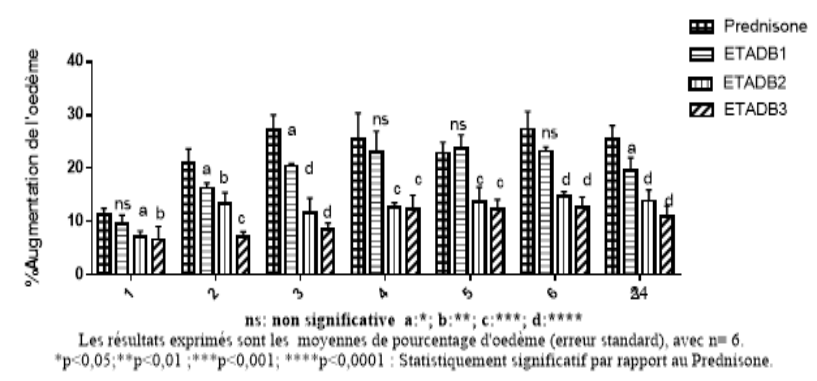

Figure 5. Effet d'augmentation de l'œdème par l'extrait et le Prednisone

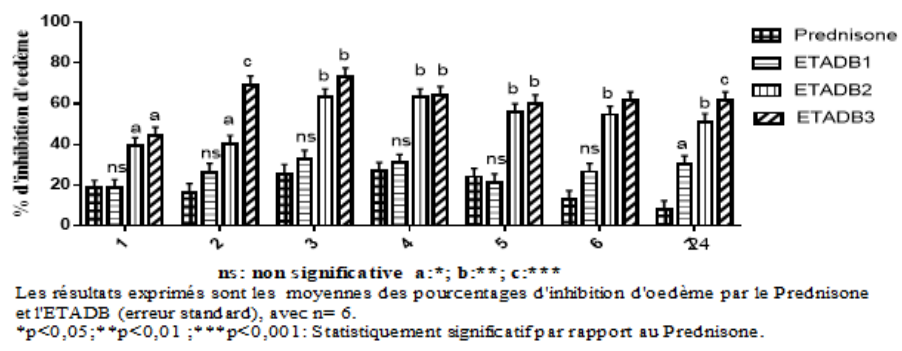

Figure 6. Effet d'inhibition de l'œdème par l'extrait et le Prednisone

\section{Effet anti-inflammatoire sur l'expression des leucocytes et des plaquettes}

Dans la présente étude, nous nous intéresserons aux leucocytes et aux plaquettes qui sont des marqueurs de l'inflammation sérique (Figure 7 et 10). L'injection des produits de Dichlorofenac, de Prednisone et de l'extrait aqueux de D. benthamianus a entrainé une baisse significative $(\mathrm{p}<0.01)$ du taux de leucocytes dans le sang par rapport aux témoins œdémateux $(13.19 \pm 0.73$ $10^{3} / \mathrm{mm}^{3}$ ). Le taux de leucocytes des rats traités avec les différents produits ne sont pas significativement différents $(p>0.05)$ du taux de leucocytes obtenus chez les rats témoins sains $(9.18 \pm 0.74)$. Les taux de neutrophiles $(6.19 \pm 0.31$ $\left.10^{3} / \mathrm{mm}^{3}\right)$ et de monocytes $\left(1.58 \pm 0.2410^{3} / \mathrm{mm}^{3}\right)$ des rats œdémateux sont significativement supérieurs $(\mathrm{p}<0.01, \mathrm{p}<0.05$ respectives $)$ à ceux des rats témoins $\left(3.88 \pm 0.37 \quad 10^{3} / \mathrm{mm}^{3}\right.$ de neutrophiles et $1.13 \pm 0.13 \quad 10^{3} / \mathrm{mm}^{3}$ monocytes). Par contre, il n'a pas été observé de différence significative ( $p>0.05$ ) entre les lots traités et témoin (Figure 8 et 9).

Les taux de plaquettes des lots témoins œdémateux $(763 \pm 21.26$ $\left.10^{3} / \mathrm{mm}^{3}\right)$ et les rats traités à la prednisone $\left(721 \pm 27.7210^{3} / \mathrm{mm}^{3}\right)$ sont très significativement élevés par rapport à celui des rats témoins sains. Celui des rats du lot EADB1 $\left(683 \pm 23.2910^{3} / \mathrm{mm}^{3}\right)$, est significativement supérieur $(\mathrm{p}<0.01)$ à celui du lot témoin sain qui est de $515 \pm 27.7210^{3} / \mathrm{mm}^{3}$. Cependant il n'existe pas de différence significative ( $p>0.05)$ entre les taux plaquettaires enregistrés au niveau des lots témoins sains et les lots EADB2 $(646.7 \pm 7.0$ $\left.10^{3} / \mathrm{mm}^{3}\right)$, EADB3 $\left(563 \pm 30.2710^{3} / \mathrm{mm}^{3}\right)$ et de Diclofenac $(561 \pm 23.3$ $\left.10^{3} / \mathrm{mm}^{3}\right)$. 


\section{Evaluation de la CRP sérique après induction de l'œè̀me par la carragénine.}

Les résultats du dosage de la C-Réactive Protéine (CRP) sont représentés dans la figure 11. Ces résultats montre qu'il existe une différence significative entre la concentration en CRP des rats témoins œdémateux et celles des témoins sains $(\mathrm{p}<0.0001)$. La CRP des lots de Diclofenac et de prednisone est significativement élevée par rapport aux témoins sains $(\mathrm{p}<0.05$ et $\mathrm{p}<0.01$ respectives).

La comparaison entre la teneur sérique en CRP des lots traités par l'extrait aqueux de D. benthamianus révèle une différence négligeable par rapport aux témoins sains. En revanche, il existe une différence significative entre les lots traités par la plante et le témoin œéémateux $(\mathrm{p}<0.05$ pour l'EADB1 et $\mathrm{p}<0.01$ pour EADB2 et EADB3).

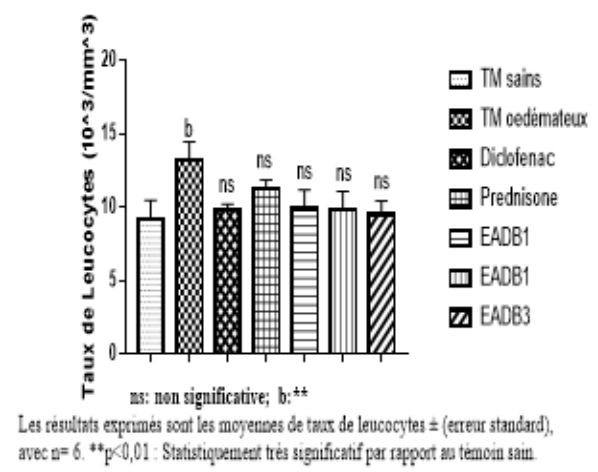

Figure 7. Taux de leucocytes

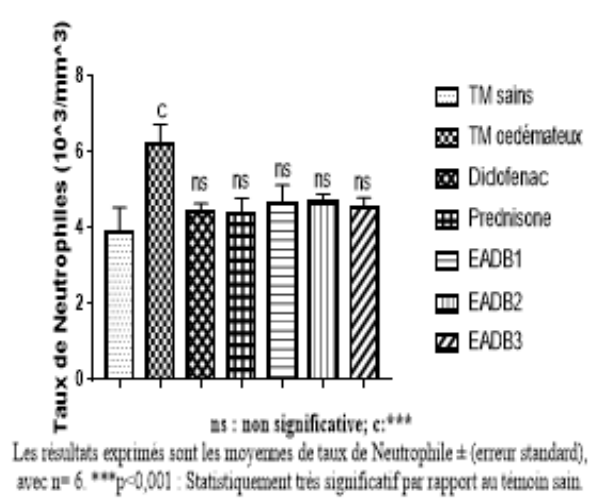

Figure 9. Taux de neutrophiles

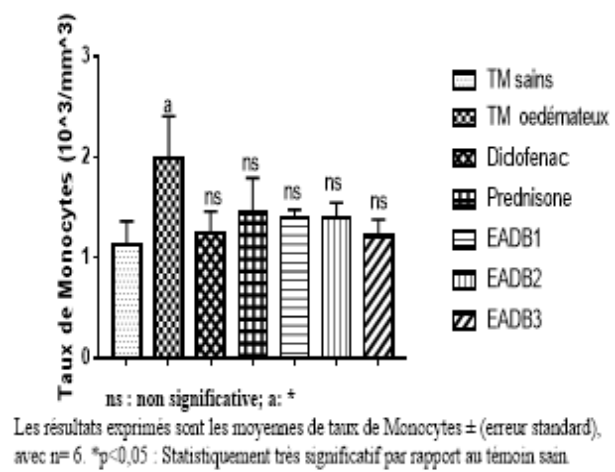

Figure 8. Taux de monocytes

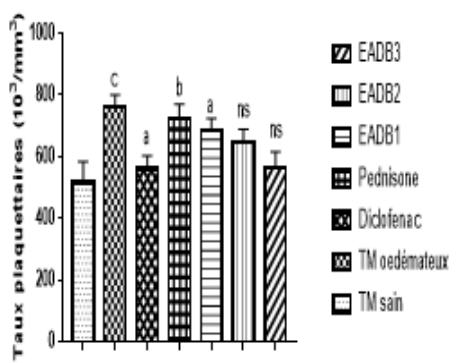

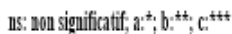

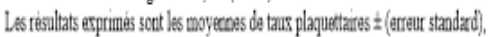

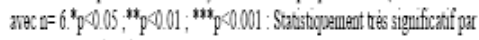
rapport autenoin sain

Figure 10. Taux de plaquettes sanguin 


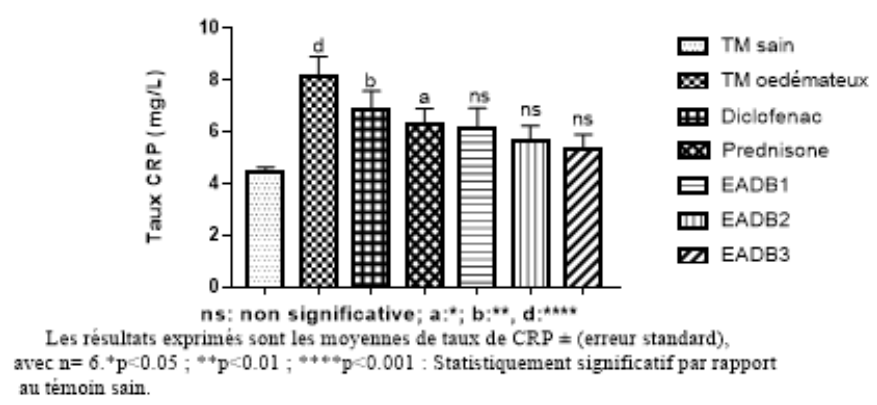

Figure 11. Taux de la Concentration de Protéine Réactive (CRP)

\section{Discussion}

\section{Etude phytochimique}

L'étude phytochimique basée sur les réactions en tubes, ont révélé que l'extrait aqueux de Distemonanthus benthamianus renferme la plupart des groupes chimiques recherchés à savoir les polyphénols, les flavonoïdes, les tanins galliques, les saponines, les coumarines, les alcaloïdes. Ces résultats, confirmés à travers les CCM sont en accord à ceux de Obame (2009) et Akapo et collaborateurs (2020), qui en plus de ces composés, a mise en évidence d'autres composés tels que les composés réducteurs et les protoantocyanes qui n'ont été recherché dans cette étude.

Ces molécules sont impliquées dans la gestion de plusieurs maladies telles que les tumeurs, les cancers, le diabète, les inflammations, la drépanocytose et le stress oxydatif (Guerrero et Ruter, 2002 ; Ndukwe et al., 2005 ; Arisawa et al., 2006; Sahu et Saxena, 2013; Kplé et al., 2020).

L'œdème induite par l'ingestion de la carragénine au niveau de l'aponévrose plantaire de la patte postérieure est l'examen le plus fréquemment adopté pour évaluer l'activité anti-inflammatoire des produits naturels (Ouedraogo et al., 2012). La carragénine provoque une inflammation typiquement liée à l'activation de la cyclooxygénase (Di Rosa, 1972). Le développement de l'œdème après son injection, est un processus biphasique (Wantana et al., 2009). La première phase se produit juste après son administration, entrainant la libération de l'histamine et la sérotonine à partir des mastocytes. Ces facteurs provoquent des modifications vasculaires qui conduisent à l'exsudation plasmatique (Subhan et al., 2007 ; Akinedele et Adeyni, 2007). Elle est suivie dans la seconde, par un gonflement, causé par la libération accrue des métabolites de l'acide arachidonique tels que les prostaglandines et les leucotriènes dans la zone inflammatoire (Yam et al., 2009 ; Buapool et al., 2013 ; Mandal et al., 2015). Cette étape est sensible aux antagonistes de synthèses des prostaglandines et aux anti inflammatoires naturels ou de synthèses tels que les glucocorticoïdes (Mhadhebi et al., 2011 ; Reto \& al., 
2014). Les anti-inflammatoires non stéroïdiens également inhibent les enzymes de la cyclooxygénase impliquées dans la synthèse de prostaglandines (Kulkani et al., 2000).

\section{Evaluation de l'activité anti-inflammatoire}

L'évaluation de l'activité anti-inflammatoire de la plante a été évaluée en suivant l'évolution des œdèmes, le dosage de la CRP et la NFS. Dans cette étude, il a été question d'abord de vérifier la validité de la méthode en testant le Diclofénac, un anti-inflammatoire non stéroïdien et le prednisone (un antiinflammatoire stéroïdien) efficaces contre l'œdème à la carragénine (Ravi et al., 2011).

L'augmentation des diamètres des pattes lésées jusqu'à la 6ème heure chez les témoins œdémateux, indique que toute réduction d'œdème durant les 6 heures serait due aux effets des produits administrés. Durant cette étude cinétique, le Diclofénac de Sodium a réduit l'augmentation de l'œdème dès la première heure avec $33.99 \%$ d'inhibition. Il a inhibé l'œdème pendant tout le temps de l'expérimentation et a montré un pourcentage d'inhibition maximal de $52.88 \%$ à la 3ème heure. Par contre, le Prednisone a réduit faiblement l'œdème dès la première heure avec un pourcentage d'inhibition de $18.57 \%$ et a atteint son activité maximale à la 4ème heure avec un pourcentage d'inhibition de $26.66 \%$. Concernant l'extrait, il a plus ou moins réduit l'œdème déjà à la lère heure, avec respectivement des pourcentages d'inhibition les plus élevés $39.01 \%$ et $44.16 \%$ aux doses 400 et $800 \mathrm{mg} / \mathrm{kg} / \mathrm{pc}$. L'extrait tout comme le Diclofénac, a atteint son activité maximale à la 3ème heure avec une inhibition de $44.95 \%$; $60.03 \%$ et $70.19 \%$ respectivement aux doses 200,400 et $800 \mathrm{mg} / \mathrm{kg} / \mathrm{pc}$. L'activité inhibitrice de l'extrait aqueux de D. benthamianus sur la période d'expérimentation était tout à fait comparable à celle du Diclofénac de sodium et non à celle de la prednisone. L'activité maximale de la plante étant observé à la 3ème heure d'expérimentation au moment de la libération des prostaglandines dans le site inflammatoire, pourrait s'expliquer par la présence au sein de cette plante, des inhibiteurs de la cyclooxygénase qui conduiraient à l'inhibition de la synthèse des prostaglandines. Ce résultat confère à la plante un mécanisme d'action anti inflammatoire semblable à celui des anti-inflammatoires non stéroïdiens et pourrait s'expliquée par la présence des composés bioactifs tels que les composés phénoliques et les saponines (Santengelo, 2007). De nombreuses études ont montré que les polyphénols et leurs métabolites inhiberaient les activités enzymatiques du métabolisme de l'acide arachidonique et réduiraient la production des médiateurs de l'inflammation tels que l'acide arachidonique, des monoxydes d'azote, de prostaglandines et de leucotriènes (Kim et al., 2004 ; Guo et al., 2009). Les polyphénols sont donc responsables des activités anti-inflammatoires d'où leur utilisation comme des agents chimio préventifs 
potentiels (Sahu et Saxena, 2013). L'effet anti inflammatoire des stérols, terpènes et saponines pourrait être dû à une inhibition de la cyclooxygénase et la libération des cytokines pro-inflammatoires (Patocka, 2003 ; Awad et al., 2005 ; Araico et al., 2007).

Les résultats obtenus dans ce test permettent d'affirmer aussi que l'activité anti-inflammatoire des extraits de $D$. benthamianus est liée en grande partie à l'effet des extraits sur l'infiltration des cellules inflammatoires ainsi que leurs médiateurs libérés dans le site inflammatoire. Pour cela, la migration cellulaire et la production de certains médiateurs clés de l'inflammation sont vérifié dans la présente étude.

\section{Réaction du produit sur leucocytes du le sang}

La NFS (numération de la formule sanguine) a été utilisé dans cette étude afin de quantifier le taux de leucocytes et principalement les monocytes, les neutrophiles et les lymphocytes dans le sang des rats. En effet, lors de l'inflammation induite par la carragénine, les monocytes et les neutrophiles sont recrutés depuis le sang vers le lieu du désordre pour y remplir leurs fonctions de phagocytes (Kumar et Robbins, 2007 ; Kumar et al., 2013). Les analyses hématologies ont indiquées un taux élevé de ces cellules chez les rats œdémateux non traités par rapport aux lots traités et témoins sains.

Les produits administrés ont réduit le taux des leucocytes. En effet l'administration des anti-inflammatoires entraine une baisse du taux de leucocytes dans le sang. Par ailleurs, les polyphénols, flavonoïdes et tanins sont de meilleurs inhibiteurs de la migration des leucocytes (Middleton et al., 2009), ceci pourrait justifier ces résultats. De même, la NFS a indiqué que des lots traités avec le $\mathrm{D}$. benthamianus ont un taux plaquettaire très proche du témoin sain, cette baisse serait liée à la baisse de production des molécules pro-inflammatoires. En effet, Les thrombocytes en plus de leur principale fonction d'agrégation plaquettaire pendant la coagulation sanguine (Fujimori et al., 1998) ont aussi la capacité de reconnaître les corps étrangers, d'initier et de moduler des réponses inflammatoires (Klinger, 1997). Lors des phénomènes inflammatoires, les médiateurs inflammatoires libérés entrainent une production accrue de plaquettes (Weill et al, 2003 ; Esmon, 2005). L'extrait agirait dont sur ces médiateurs en inhibant leur synthèse, ce qui justifie la différence significative de taux plaquettaire entre les rats œdémateux traités et les rats non traités.

\section{Réaction du produit sur le taux de la CRP sanguine}

En ce qui consterne le taux de CRP présente dans le sérum des rats, la carragénine a provoqué une augmentation de la CRP chez tous les rats. Cette différence significative chez les témoins odémateux serait due à la synthèse et à la forte libération de la CRP dans le sang sous l'influence des médiateurs 
de l'inflammation induite par la carragénine (Jialal et al., 2004 ; Ablij, 2002). L'administration de l'ETA de $D$. benthamianus et des molécules de référence aux rats œdémateux a diminué la concentration de CRP induit par l'injection de la carragénine. Par ailleurs, la présence des groupes chimiques dans la plante serait responsable de l'inhibition de la synthèse des molécules proinflammatoires responsables de la forte libération des protéines de l'inflammation (CRP, haptoglobine, orosomucoïde, céruléoplasmine) lors des phénomènes inflammatoires (Jialal et al., 2004 ; Giraudet, 1992).

\section{Conclusion}

Cette étude s'inscrit dans le cadre de la valorisation des plantes médicinales et surtout de la recherche de nouvelles molécules à partir d'extraits de plantes. Au cours de ce travail, Les études phytochimiques basée sur des tests spécifiques ont montré la présence des polyphénols, des flavonoïdes, des saponines, des alcaloïdes, des poly terpènes et des stérols dans l'extrait aqueux des écorces de $D$. benthamianus. Cette plante représente une source potentielle de molécule bioactive. Par ailleurs, l'activité antiœdémateuse de la plante a permis de confirmer les propriétés antiinflammatoires de la plante puisqu'il réduit de façon significative les œdèmes des pattes des rats. Cette activité est comparable à celle de diclofénac qui est un antiinflammatoire de référence. Ceci a été aussi confirmé par l'étude sérologique et hématologique qui a montré une augmentation significative des globules blancs, des plaquettes, de CRP pour les rats témoins œdémateux par rapport aux rats traitées par l'extrait.

L'activité anti-inflammatoire des écorces de $D$. benthamianus montre que cette plante possède un puissant effet thérapeutique ce qui supporte son usage traditionnel pour le soulagement de divers affections inflammatoires. Les résultats obtenus lors de cette étude sont intéressants, cependant des études complémentaires seront nécessaires afin de comprendre les mécanismes cellulaires et moléculaires liés à cette activité antiinflammatoire. Ces études doivent être orientées vers la détermination des composés actifs de Distemonanthus benthamianus et l'évaluation de leurs effets sur le processus inflammatoire.

\section{References:}

1. Ablij, H. (2002). C-reactive protein : history and revival. Eur. J. Intern. Med. 13(7):412-22.

2. Aiyegoro, O.A., Akinpelu, D.A., Afolayan, A.J. \& Okoh, A.I., (2008). Antibacterial activities of crude stem bark extracts of Distemonanthus benthamianus Baill. Journal of Biological Sciences 8(2): 356-361.

3. Akakpo-Akue, J., Kplé, T.K.M., Coulibaly, K., AHON, G.M., Fofié, Y., Yapo -Crezoit. A., Zirihi, G.N. \& Kra, A. K.M. (2020). 
Ethnobotanical study of medicinal plants used against sickle cell anaemia in the eastern part of the Côte d'Ivoire; J.Anim.Plant Sci. Vol. 45 (1): 7839-7852.

4. Akendengué, B. \& Louis, A.M. (1994). Medicinal plants used by the masango people in gabon. Journal of Ethnopharmacology 41, 193200.

5. Akindele, A.J. \& Adeyemi, O. (2007). Antipyretic activity of Byrsocarpus coccineus Schum and Thonn. Internat J Pharmaocol, 4: 357-612.

6. Anupama, A. S., Kishor, N. R., Rahul, D. K., et Kanchan, S. M. (2012). Evaluation of antiinflammatory and analgesic activities of Tamarindus indica seeds. Int J Pharm Sci Drug Res; 4(3): 213-217.

7. APG IV. (2016). An update of Angiosperm Phylogeny Group classification for the orders and families of flowering. Botanical Journal of the Linnean Society. 181: 120.https://doi.org/10.1111/boj.12385.

8. Araico, A., Terencio, M. C., Alcaraz, M. J., Domínguez, J. N., León, C. \& Ferrándiz, M. L. (2007). Evaluation of the antiinflammatory and analgesic activity of Me-UCH9, a dual cyclooxygenase2/5lipoxygenase inhibitor. Life Sciences ; 80: 2108-2117.

9. Arisawa, K., Soda, M. \& Akahoshi, M. (2006). Human T-cell lymphotropic virus type-1 infection and risk of cancer : 15.4 year longitudinal study among atomic bomb survivors in Nagasaki, Japan. Cancer Sci, 97, 535-9.

10. Awad, E.M. (2005). In vitro decrease of the fibrinolytic potential of cultured human fibrosarcoma cell line, HT1080, by Nigella sativa oil. Phytomedicine; 12: 100-107.

11. Békro, Y.A., Mamyrbekova, B.J.A., Boua, B.B., Tra Bi F.H. \& Ehilé E.E. (2007). Etude ethnobotanique et screening phytochimique de Caesalpinia benthamiana (Baill.) Herend. Et Zarucchi (Caesalpiniaceae). Sciences et Nature 4 (2) : 217-225.

12. Brou, K.G., Mamyrbekova-Békro, J., Dogbo, D.O., Gogbeu, S.J. \& Békro, Y.A. (2010). On the Qualitative Phytochemical Composition of Crude Hydromethanolic extracts of the Leaves of 6 Varieties of Manihot Esculenta Crantz of Côte d'Ivoire. European Journal of Scientific Research 45(2): 200-211.

13. Buapool, D., Mongkol, N., Chantimal, J., Roytrakul, S., Srisook, E. \& Srisook, K. (2013). Molecular mechanism of anti-inflammatory activity of Pluchea indica leaves in macrophages RAW 264.7 and its action in animal models of inflammation. J. Ethnopharmacol. 146, 495-504. 
14. Bukhari (2013). The central analgesic and anti-inflammatory activities of the methanolic extract of carthamus oxycantha; journal of physiologyand, 64, 3, 369-375.

15. Cheriti, A., Rahmani, S. \& Belboukhari, N. (2016). Evaluation de l'activité antiinflammatoire d'extraits aqueux de feuilles Limoniastrum feei (Plumbaginacea). Algerian Journal of Arid Environment "AJAE", 6(1), 80-86.

16. Chiolero, A., Würzner, G. \& Burnier, M. (2000). Les inhibiteurs sélectifs de la cyclooxygénase de type $2:$ moins d'effets rénaux que les anti-inflammatoires non stérö̈diens classiques. Néphrologie, 21 : 425-430.

17. CIRAD Forestry Department, (2009). Movingui. [Internet] Tropix 6.0. http://tropix.cirad.fr/ africa/ movingui.pdf. March 2012.

18. Di Rosa, M. (1972). Biological properties of carrageenan. J Pharm Pharmacol; 24: 89-102.

19. Distemonanthus benthamianus (PROTA). (2019). PlantUseFrançais. https://uses.plantnetproject.org/fr/Distemonanthus_benthamianus_(P ROTA).

20. Esmon (2005). The interaction between Inflammation and coagulation. Br. J. Haematol, 131:417-430.

21. Fujimori, H., Ozaki, K. \& Nomuras, S. (1998). Characterisation of platelets abnormalities of Tester Moriyama (TM) rats with storage pool deficiency. Lab. Anim. Sci, 48, $5:$ 490-495.

22. Giraudet P. (1992). Concept et intérêt clinique des profils protéiques, Feuillets Bio ; 33/188 : 61-69.

23. Guerrero, J.M. \& Reiter, R.J. (2002). Melatonin-immune system relationships. Curr Top Med.

24. Guo, W., Kong, E. \& Meydani, M. (2009). Dietary Polyphenols, Inflammation and Cancer. Nutritionandcancer,61 (6): 807-810.

25. Jialal, I., Devaraj, S. \& Venugopal, S.K. (2004). C-Reactive Protein: Risk Marker or Mediator in Atherothrombosis. Hypertension, 44: 611.

26. Kulkami, S.K., Jain N.K. \& Singh A. (2000). Cyclooxygenase isoenzymes and newer therapeutic potential for selective COX-2 inhibitors. Methods and Findings in Expermental and Clinical Pharmacology, 22:291-298.

27. Kumar, S. \& Pandey, A.K., 2013. Chemistry and biological activities of flavonoids: an overview. The Scientific World Journal, ID: 162750; $1-16$.

28. Kumar, V., \& Robbins, S.L. (2007). Robbins basic pathology (Philadelphia, PA: Saunders/Elsevier). 
29. Kim, H.P., Son K.H. \& Chan H.W., 2004. Anti-inflammatory plant flavonoids and cellular action mechanisms. J Pharmacol Sci; 96 : 229245.

30. Kple, T.K.M., Akakpo-Akue, J., Golly, J.K., Fofié, Y., Ahon, M.G., Kra, M.A., Sanogo, I. \& Yapo-Crezoit, A.C. (2020). Phytochemical characterization of three plants and their antisickling activity in the management of sickle cell disease; Journal of Bosciences and Medecine, V8: 100-112.

31. Klinger, M.H. (1997). Platelets and inflammation. Anat Embryol; 196 : 1-11.

32. Mandal, G., Chatterjee, C. \& Chatterjee, M. (2015). Evaluation of antiinflammatory activity of methanolic extract of leaves of Bougainvillea spectabilis in experimental animal models. Pharmacolognosy Res. JanMar; 7(1): 18-22.

33. Middleton S., Vermeulen W., Byth Wilson K., Sullivan C. \& Middleton, P. 2009. Treatment of obstructive sleep apnoea in Samoa progressively reduces daytime blood pressure over 6 months. Respirology, 14(3), 404-410.

34. Mhadhebi, L., Laroche-Clary, A., Robert, J. \& Bouraoui, A. (2011). Antioxidant, anti-inflammatory, and antiproliferative activities of organic fractions from the Mediterranean brown seaweed Cystoseira sedoides Revue canadienne de physiologie et pharmacologie, 89 (12): 911-921

35. Nguelefack, E.M.P., Ngu, K.P., Atchade, A., Dimo, T., Tsabang, N. \& Mbafor, J.T. (2005). Phytochemical composition and in vitro effects of the ethyl acetate bark extract of Distemonanthus benthamianus Baillon (Caesalpiniaceae) on Staphylococcus aureus and Streptococcus agalactiae. Cameroon J. Exp. Biol., 1: 50-53.

36. Ndukwe, K.C., Okeke, I.N., Lamikanra, A., Adesina, S.K. \& Aboderin, O. (2005). Antibacterial activity of aqueous extract of selected chewing sticks. J. contemp. Dent. Pract; 3(6):86-94.

37. Ouédraogo, N., Lompo, M., Sawadogo, R.W., Tibiri, A., Hay, A.E., Koudou J. \& Guissou I. P., (2012). Etude des activités antiinflammatoire, analgésique et antipyrétique des décoctés aqueux des feuilles et des racines de Pterocarpus erinaceus Poir. (Fabaceae). Phytothérapie. pp. 1-7.

38. Patocka, J. (2003). Biologically active pentacyciic triterpenes and their current medicine signification. J. Appl. Biomed 1, 7-12.

39. Ravi S.S., Mushtaq, A., Zahoor, A.W., Vikas, S., Vijay, V.M., \& Prerna, U., (2011). Antiinflammatory effects of Dashmula, an Ayurvedic preparation, versus Diclorofenac in animal models. J Chem Pharm Res; 3: 882-888. 
40. Reto, M., Almeida, C., Rocha, J., Sepodes, B., \& Figueira, M.E., (2014). Green Tea (Camellia sinensis): Hypocholesterolemic Effects in Humans and Anti-Inflammatory Effects in Animal. Pharmacology \& Pharmacy; 5: 1113-1118.

41. Sahu, R. \& Saxena, J. (2013). Screening of Total Phenolic and Flavonoid Content in Conventional and Non-Conventional Species of Curcuma. Journal of Pharmacolognosy and Phytochemitry, 2(1): 176179.

42. Santangelo, C., Varì, R., Scazzocchio, B., Di Benedetto, R., Filesi, C., \& Masella, R. (2007). Polyphenols, intracellular signalling and inflammation. Annali-Istituto Superiore Di Sanita, 43 (4), 394.

43. Soubrier, M., Rosenbaum, D. \& Tatar, Z. (2013). Antiinflammatoires non stéroïdiens et vaisseaux. Revue du rhumatisme, 80(3), 204-208.

44. Subhan, N., Alam, A., Ahmed, F. \& Shahid, I.Z. (2008). Antinociceptive and gastroprotective effect of the crude ethanolic extracts of Excoecaria agallocha linn. Turk J. Pharm. Sci. 5 (3): 143154.

45. Trease, G. \& Evans, S.M. (2002). Pharmacognosy. 15th Ed. English Language Book Society, Bailliere Tindall, London, pp 23-67

46. Viladomiu, M., Hontecillas, R. \& Bassaganya-Riera J. (2016). Modulation of inflammation and immunity by dietary conjugated linoleic acid. European journal of pharmacology, 785, 87-95.

47. Wantana, R., Tassanee, N. \& Subhad-hirasakul, S. (2009). Antinociceptive, antipyretic, and anti-inflammatory activities of Putranjiva roxburgthii Wall. Leaf extract in experimental animals. J Nat Med, 63 (3): 27 -

48. Weill, B., Bateux, F. \& Dhainaut, J. (2003). Immunopathologie et reactions inflammatoires. Eds, De Boeck, Universite (Paris) ; 12-23.

49. Winter, C.A., Risley, F.A. \& Nuss, O.W. (1962). Carrageenin induced oedema in hand paw of the rat as assays anti-inflammatory drugs. Proc. Soc. Exp. Biol. Med., 111, 544-547

50. Yam, M.F., Ang, L.F., Ameer, O.Z., Salman, I.M., Aziz, H.A. \& Asmawi, M.Z. (2009). Anti-inflammatory and Analgesic Effects of Elephantopus tomentosus Ethanolic Extract; J.Acupunct Meridian Stud; 2 ( 4):280-287 